\title{
Design of Circularly Polarized Transmit array Antenna using Low-Profile Dual-Linearly Polarized Elements
}

\author{
N.Khadarbasha ${ }^{1} \&$ A.Yogeshwaran ${ }^{2}$ \\ ${ }^{1,2}$ Assistant Professor, Dhanalakshmi Srinivasan Engineering College, Perambalur, India.
}

DOI: http://doi.org/10.38177/ajast.2020.4404

Copyright: $\odot 2020$ N.Khadarbasha et al. This is an open access article distributed under the terms of the Creative Commons Attribution License, which permits unrestricted use, distribution, and reproduction in any medium, provided the original author and source are credited.

\section{ABSTRACT}

A low-profile dual-linearly-polarized unit cell in X-band, and its capability is demonstrated by a circularly polarized transmit array. The unit cell comprises three metallic layers etched on two dielectric slabs without air gap. Cross strips are inserted in cross slots on the top and bottom layers, and the T-slot structure is etched on the middle layer. The proposed unit cell has high isolation between the dual polarizations, and its total thickness of the unit cell is only $1 \mathrm{~mm}$. Prototype of a 341-element transmit array, the incoming incident linearly polarized wave is transformed into the outgoing circularly polarized wave, is simulated. The measured results show that the proposed transmit array realizes $3.5 \%(9.8-10.15 \mathrm{GHz})$, axial ratio bandwidth and $4 \%(9.7-10.1 \mathrm{GHz}) 1-\mathrm{dB}$ gain bandwidth. The measured peak gain at $10 \mathrm{GHz}$ is $21.9 \mathrm{dBi}$, with the aperture efficiency of $36 \%$.

Keywords: Low profile, Dual polarization, Transmitarray.

\section{Introduction}

The transmit array antenna has attracted significant attention in past decades because of its advantages such as high gain, inexpensive fabrication, and no insertion loss caused by the feeding network of a phased array at higher frequencies. With simple planar structure, the transmit array has more advantages over lens antennas. In addition, compared with the reflect array, in the transmit array the feed is placed directly in back of the aperture to avoid its blockage losses, which usually results in higher aperture efficiency. For the transmit array, a feed antenna illuminates the array in which the proper designed unit cells are used to transform the incoming wave with proper phase to form a desired beam shape.

Two most important design criteria for a transmit array element are its phase range and transmission magnitude. In order to obtain a transmit array with high aperture efficiency, unit cells with $360^{\circ}$ transmission phase range and the transmission magnitude as high as possible are needed. To date, many different efforts have been devoted to design the transmit arrays with high performance.

To date, many different efforts have been devoted to design the transmit arrays with high performance. A wideband transmit array antenna using four identical layers with the double loop elements is proposed [2]. A transmitarray antenna consists of multiple layers of flat transmitting surface and an illuminating feed source, which is located on an equivalent focal point.

The transmission coefficients of these elements are individually designed such that spherical phase front from the feed source is converted into a plan phase front. One of the arrays act as a receiver, and it is illuminated by the antenna feed source. The other array radiates into free space, and it acts as a transmitter. A coupling structure is designed between the two array to obtain a specific phase and magnitude distribution. It is worthwhile to point out that these techniques mostly use printed type elements mounted on substrate materials [3],[4]. 


\section{Proposed System}

In transmitarrays, at least three layers with quarter-wavelength spacings are necessary to meet a full $360^{\circ}$ phase range requirement [13]. In our study, dual linear polarizations, low profile and a full $360^{\circ}$ phase range are important for the transmitarray element design. The designed transmitarray unit cell comprises of three metallic layers sandwiched with two dielectric layers. Its configuration is shown in Figure 1. The substrate layers are made by RO4003 with a relative permittivity of 3.55 and a thickness of $\mathrm{H}=0.5 \mathrm{~mm}$.

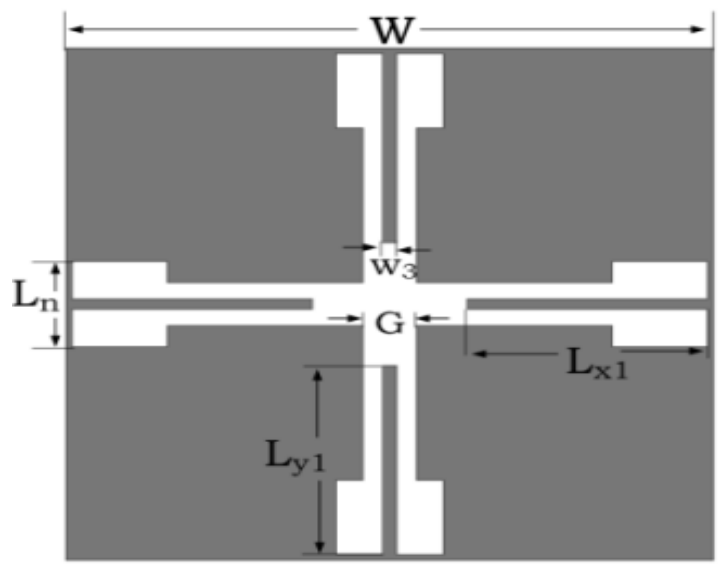

Figure 1: Unit Cell Configuration of T-Slot Element

Table 1

PARAMETER VALUES OF THE UNIT CELL

\begin{tabular}{|c|c|c|c|}
\hline Parameters & Values (mm) & Parameters & Values (mm) \\
\hline $\mathrm{W}_{1}$ & 0.4 & $\mathrm{G}$ & 0.7 \\
\hline $\mathrm{W}_{2}$ & 0.2 & $\mathrm{Lx}$ & vary \\
\hline $\mathrm{L}_{1}$ & 9 & $\mathrm{Ly}$ & vary \\
\hline $\mathrm{Ln}$ & 1.6 & $\mathrm{Lx} 1$ & $2.9+0.15^{*} \mathrm{Lx}$ \\
\hline $\mathrm{W}_{3}$ & 0.3 & $\mathrm{Ly} 1$ & $2.9+0.15^{*} \mathrm{Ly}$ \\
\hline $\mathrm{W}$ & 9.6 & $\mathrm{H}$ & 0.5 \\
\hline
\end{tabular}

\section{Unit Cell Design}

In transmitarrays, at least three layers with quarter-wavelength spacings are necessary to meet a full $360^{\circ}$ phase range requirement. In our study, dual linear polarizations, low profile and a full $360^{\circ}$ phase range are important for the transmitarray element design. The designed transmitarray unit cell comprises of three metallic layers sandwiched with two dielectric layers. Its configuration, the substrate layers are made by RO4003 with a relative permittivity of 3.55 and a thickness of $\mathrm{H}=0.5 \mathrm{~mm}$.

For the proposed element, parallel resonant circuits are formed by the cross slot and the cross strip on the top and bottom layers, and a transmission passband is realized. By independently adjusting lengths Lx and Ly, the transmission phase of the corresponding polarization wave can be controlled. It is very difficult to realize $360^{\circ}$ transmission phase on such a low profile structure by using three identical layers in our attempt, so we design a T-slot element shown in Fig. 1(b), which is used to form a new resonance. By conducting several times simulation experiments, we summarize the relation between Lx1 (Ly1) and Lx (Ly), as shown in Table 1. Through a rational design of the ratio of Lx1 (Ly1) to Lx (Ly), the desired phase range of $360^{\circ}$ can be realized. Values of the equivalent 
capacitors and inductors for $\mathrm{x}$-direction are not affected by the y-direction size, and vice versa. Therefore, the designed unit cell can realize good polarization isolation performance.

The unit cell simulations are carried out using the commercial software Ansoft HFSS ver. 13. The mutual coupling between identical elements is considered under periodic boundary conditions.

Transmission coefficient responses of the compound sandwich structure with different Lx's. It can be seen that with increasing Lx's, the pass band center frequencies decrease. The transmission amplitudes and phases versus Lx at the center frequency of $10 \mathrm{GHz}$ for different Ly's, when the incoming wave is x-polarized.

\section{Circular Polarization}

In electrodynamics, circular polarization of an electromagnetic wave is a polarization state in which, at each point, the electric field of the wave has a constant magnitude but its direction rotates with time at a steady rate in a plane perpendicular to the direction of the wave. In electrodynamics the strength and direction of an electric field is defined by its electric field vector. In the case of a circularly polarized wave, as seen in the accompanying animation, the tip of the electric field vector, at a given point in space, describes a circle as time progresses. At any instant of time, the electric field vector of the wave describes a helix along the direction of propagation. A circularly polarized wave can be in one of two possible states, right circular polarization in which the electric field vector rotates in a right-hand sense with respect to the direction of propagation, and left circular polarization in which the vector rotates in a left-hand sense. Circular polarization is a limiting case of the more general condition of elliptical polarization.

\section{Conversion to and from Linear Polarization}

Circularly polarized light can be converted into linearly polarized light by passing through a quarter-wave plate. Passing linearly polarized light through quarter-wave plate with axes at $45^{\circ}$ to its polarization axis will convert it to circular polarization. In fact, this is the most common way of producing circular polarization in practice.

\section{Simulation Results}

\section{Return Loss}

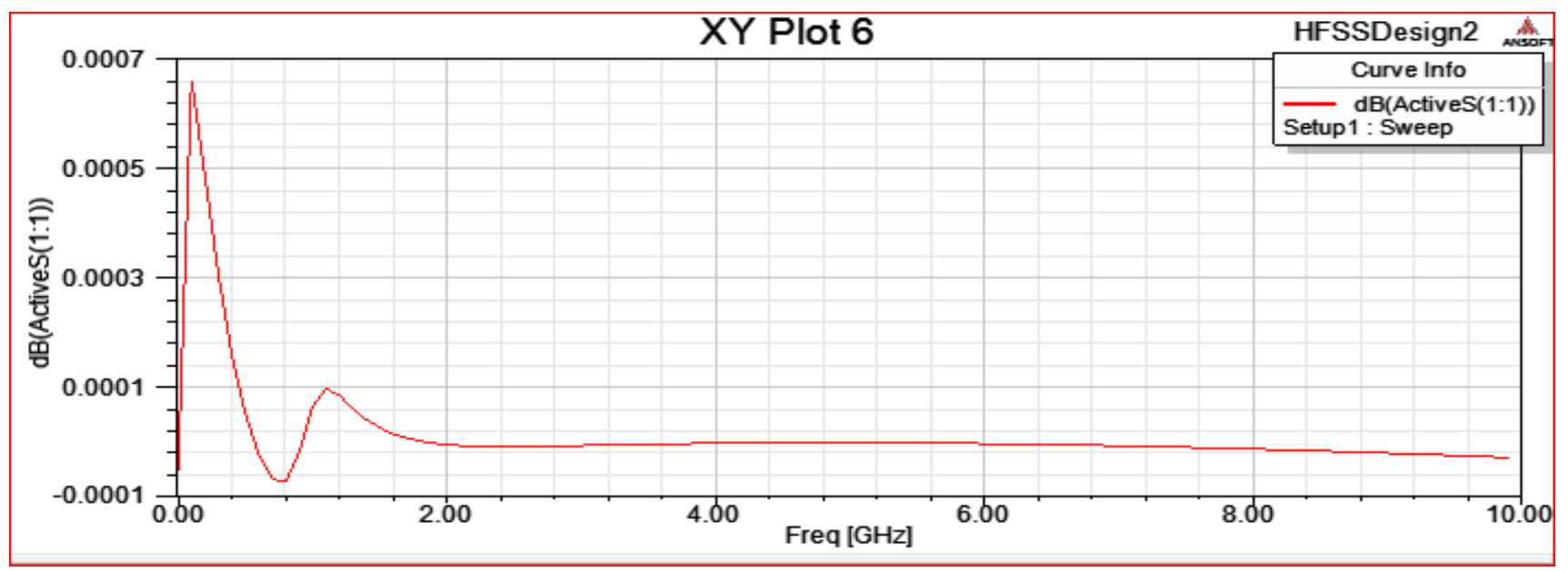

Figure 2: Return Loss of T-Slot Element 


\section{Radiation Pattern}

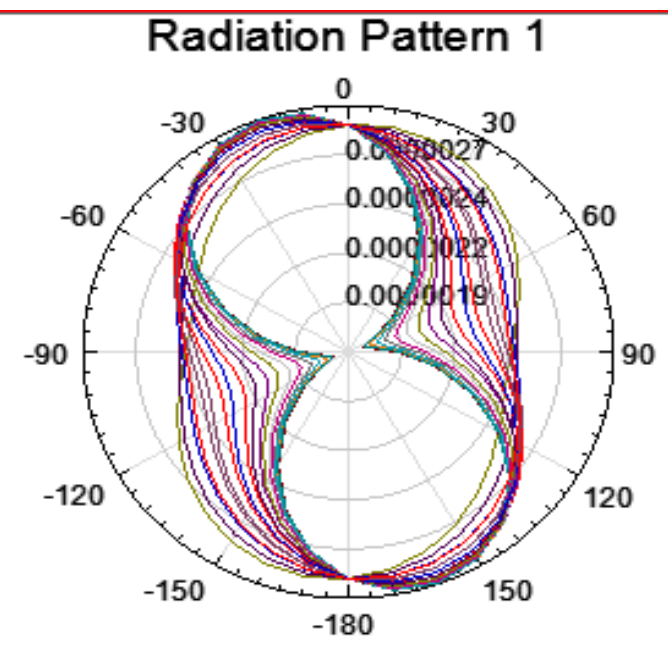

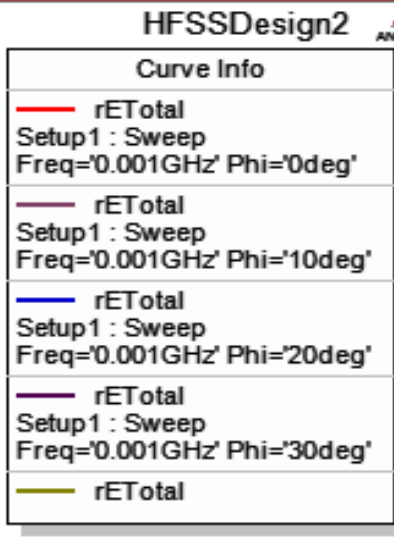

Figure 3: Radiation Pattern for Designed Transmitarray at $10 \mathrm{GHz}$

\section{Radiation Pattern 3D}

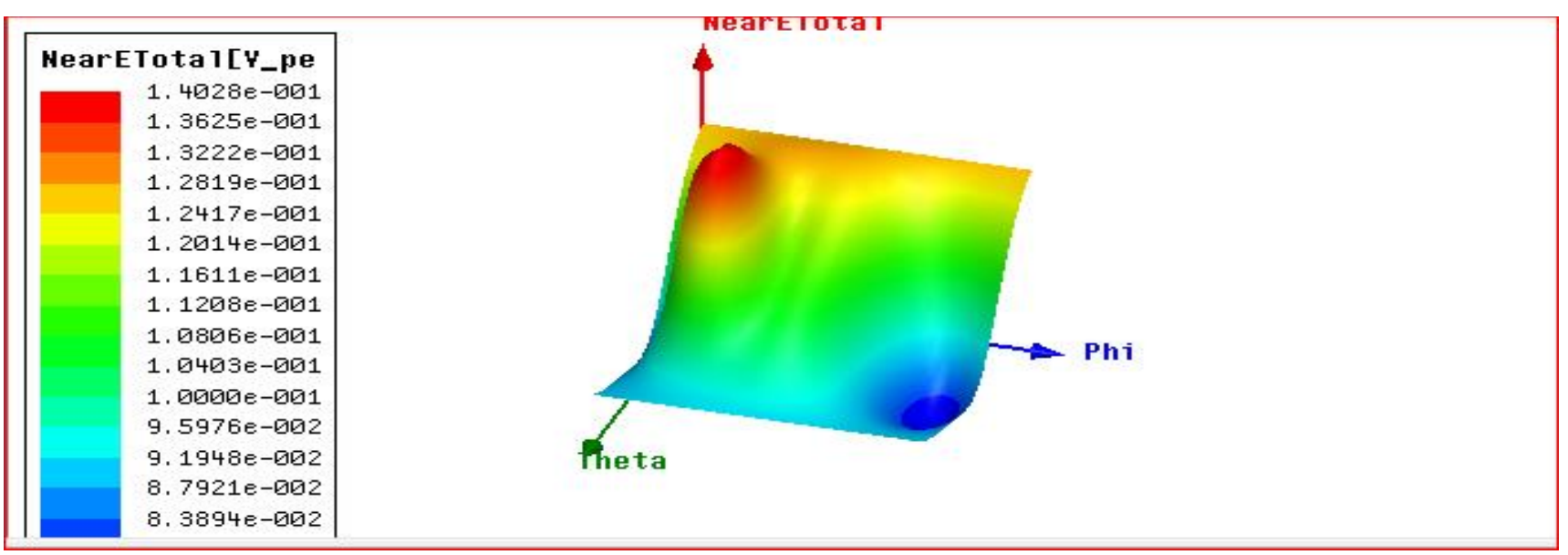

Figure 4: Radiation Pattern in 3D form of designed Transmitarray

\section{D Polar Plot}

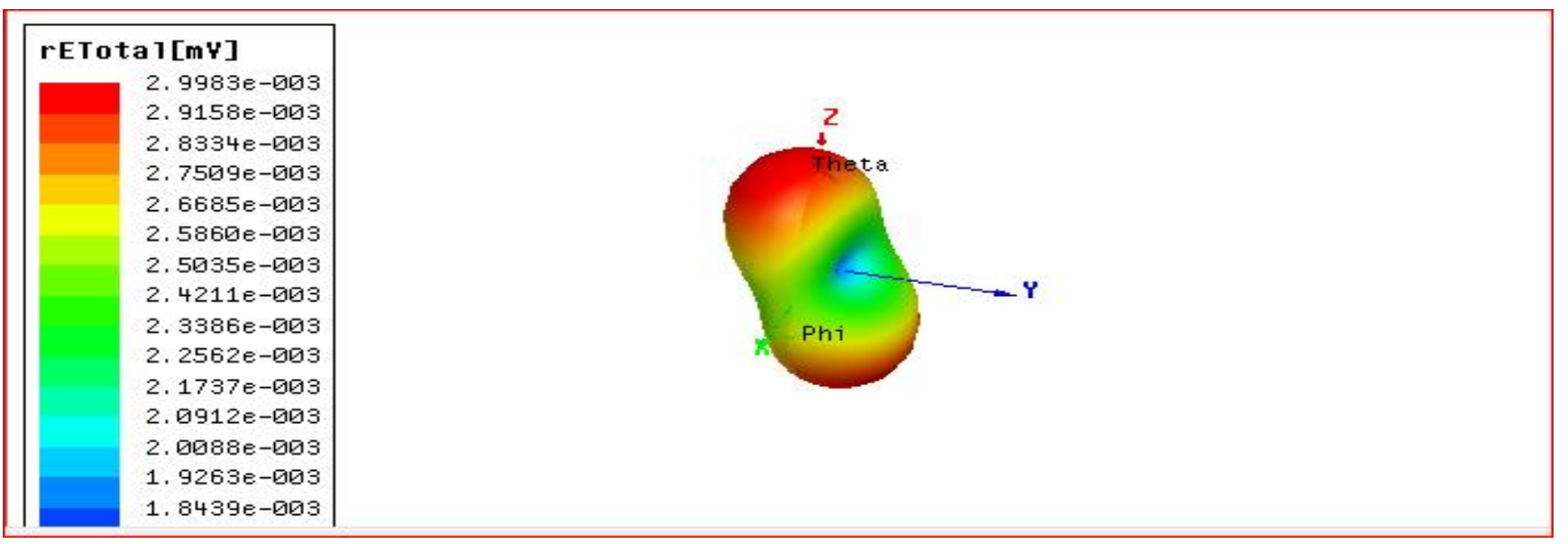

Figure 5: Polar Plot of Transmitarray Antenna 
Asian Journal of Applied Science and Technology Volume 4, Issue 4, Pages 38-43, October-December 2020

\section{Conclusion}

A circularly polarized transmit array antenna using a novel low-profile dual-polarized unit cell, that consists of three metallic layers with two thin dielectric slabs in between Cross strips are etched in cross slots on the top and bottom layers and the T-slot element is etched on the middle layer. The unit cell has the good dual polarization characteristic and it is compact, the unit cell element has complete symmetry, the polarization of transmit array is specified and its total thickness is $1 \mathrm{~mm}(0.033 \lambda 0)$. A 341-element circularly polarized transmit array realizes the 3-dB AR bandwidth of 3.5\% (9.8-10.15 GHz), and its 1-dB gain bandwidth is $4 \%$ (9.7-10.1 GHz). The aperture efficiency is $36 \%$ at $10 \mathrm{GHz}$ frequency.

\section{References}

[1] Chao Tian, Yong-Chang Jiao, "circularly polarized transmitarray antenna using low-profile dual-linearly-polarized elements," IEEE Antennas and Wireless Propagation Letters., pp.1536-1225, May 2016.

[2] C. G. M. Ryan, M. Reza, J. Shaker, J. R. Bray, Y. M. M. Antar, and A. Ittipiboon, “A wideband transmitarray using dual-resonant double square rings," IEEE Trans. on Antennas Propag., vol. 58, no. 5, pp. 1486-1493, May 2010.

[3] A. H. Abdelrahman, A. Z. Elsherbeni, and F. Yang, "Transmitarray antenna design using cross-slot elements with no dielectric substrate," IEEE Antennas and Wireless Propagation Letters, vol. 13, pp. 177-180, 2014.

[4] A. H. Abdelrahman, A. Z. Elsherbeni, and F. Yang, "High-gain and broadband transmitarray antenna using triple-layer spiral dipole elements," IEEE Antennas and Wireless Propagation Letters, vol. 13, pp. 1288-1291, 2014.

[5] B. Rahmati and H. R. Hassani, "High-efficient wideband slot transmitarray antenna," IEEE Trans. on Antennas Propag., vol. 63, no. 11, pp. 5149-5155, Nov. 2015.

[6] B. Rahmati and H. R. Hassani, “Low-profile slot transmitarray antenna," IEEE Trans. on Antennas Propag., vol. 63, no. 1, pp. 174-181, Jan. 2015.

[7] L. D. Palma, A. Clemente, L. Dussopt, R. Sauleau, P. Potier, and P. Pouliguen, "Circularly polarized transmitarray with sequential rotation in ka-band," IEEE Trans. on Antennas Propag., vol. 63, no. 11, pp. 5118-5124, Nov. 2015.

[8] C. Y. D. Sim, C. C. Chang, and J. S. Row, "Dual-feed dual-polarized patch antenna with low cross polarization and high isolation,” IEEE Trans. on Antennas Propag., vol. 57, no. 10, pp. 3321-3324, Oct. 2009.

[9] G. Zhao, Y. C. Jiao, F. Zhang, and Fu-Shun Zhang, "A subwavelength element for broadband circularly polarized reflect arrays,” IEEE Antennas and Wireless Propagation Letters, vol. 9, pp. 330-333, 2010.

[10] D. Cadoret, L. Marnat, R. Loison, R. Gillard, H. Legay, and B. Salome, "A dual linear polarized printed reflectarray using slot loaded patch elements," in Proc. Eur. Conf. Antennas Propag. (EuCAP’07), Edinburgh, UK, Nov. 2007, pp. 1-5. 
[11] R. D. Bari, T. Brown, S. Gao, M. Notter, and C. Underwood, "Dual-polarized printed S-band radar array antenna for spacecraft applications," IEEE Antennas and Wireless Propagation Letters, vol. 10, pp. 987-990, 2011. [12] T.Cai, G.Leon, X.F.Zhang, J.G.Liang, Y.Q.Zhuang, D.Liu, and H.X.Xu, "Ultra -thin polarization beam splitter using 2-D transmissive phase gradient metasurface," IEEE Antennas and Wireless Propag., vol.63, no.12, pp.5629-5636, Dec.2015.

[13] E.G.Plaza, G.Leon, S.Leredo, and F.L.Heras, "Dual polarized transmitarray lens," in Proc. Eur. Conf. Antennas Propag. (EuCAP'14), Hague, Netherlands, Apr.2014, pp.2305-2308.

[14] A.H.Abdelrahman, A.Z.Elsherbeni, and F.Yang, "Transmission phase limit of multilayer frequency-selective surfaces for transmitarray designs," IEEE Trans. on Antennas and Propag., vol. 62, no.2, pp.690-697, Feb.2014. 